\title{
Efeitos do Treinamento Resistido em Obesos: uma revisão integrativa
}

\author{
Effects of resistance raining on obese: an integrative review
}

Efectos del entrenamiento de resistencia sobre los obesos: una revisión integrativa

Priscila Taciana Barbosa Dutra ${ }^{1}$, Nayra Suze Souza e Silva ${ }^{1 *}$, Priscilla Durães de Carvalho ${ }^{1}$, Bruna Nathália Santos ${ }^{1}$, Walter de Freitas Filho ${ }^{1}$, Nayara Teixeira Gomes ${ }^{1}$, Isabel Cristina Alves Pereira ${ }^{2}$, Izabella Nascimento Arcanjoํ, Vitória Paula Dias Cruz', Sabrina Gonçalves Silva Pereira'1.

\section{RESUMO}

Objetivo: Revisar na literatura científica a respeito dos efeitos do treinamento resistido em obesos de qualquer faixa etária. Material e métodos: Trata-se de uma revisão integrativa, de caráter teórico e descritivo. Foram incluídos no estudo artigos originais (quantitativos e qualitativos) publicados até dezembro de 2018. Os artigos foram selecionados nas bases de dados PUBMED, PERIÓDICOS CAPES e LILACS. Foram utilizados os descritores musculação, treinamento e obesidade. Resultados e discussão: Obteve-se inicialmente 596 publicações, sendo 11 artigos selecionados no final para construção da revisão. A maioria dos artigos selecionados apresentaram diversos benefícios da prática do treinamento resistido em praticantes obesos, como ganho de massa magra, perda de peso e melhorias na qualidade de vida. Conclusão: Diante dos dados apresentados é possível observar os diversos benefícios para a saúde dos praticantes obesos. Esses resultados revelam a necessidade de programas de promoção e prevenção da saúde que alcancem toda população, inclusive pessoas com sobrepeso e obesidade.

Palavras-chave: Treinamento, Obesidade, Musculação.

\section{ABSTRACT}

Objective: To review in the scientific literature about the effects of resistance training in obese patients of any age group. Material and methods: This is an integrative review, of a theoretical and descriptive nature. Original articles (quantitative and qualitative) published until December 2018 were included in the study. The articles were selected in PUBMED, PERIÓDICOS CAPES and LILACS databases. The descriptors were used for bodybuilding, training and obesity. Results and discussion: 596 publications were initially obtained, 11 of which were selected at the end for the construction of the review. Most of the articles selected presented several benefits of practicing resistance training in obese practitioners, such as lean mass gain, weight loss and improvements in quality of life. Conclusion: Given the data presented, it is possible to observe the various health benefits of obese practitioners. These results reveal the need for health promotion and prevention programs that reach the entire population, including overweight and obese people.

Key words: Training, Obesity, Bodybuilding.

\section{RESUMEN}

Objetivo: Revisar en la literatura científica acerca de los efectos del entrenamiento resistido en obesos de cualquier grupo de edad. Material y métodos: Se trata de una revisión integrativa, de carácter teórico y descriptivo. Se incluyeron en el estudio artículos originales (cuantitativos y cualitativos) publicados hasta

\footnotetext{
1 Universidade Estadual de Montes Claros - UNIMONTES. Montes Claros - MG.

${ }^{2}$ Faculdades Integradas Pitágoras - FIPMOC. Montes Claros - MG. *E-mail: nayrasusy@hotmail.com

Financiamento: Coordenação de Aperfeiçoamento de Pessoal de Nível Superior (CAPES).
} 
diciembre de 2018. Los artículos fueron seleccionados en las bases de datos PUBMED, PERIÓDICOS CAPES y LILACS. Se utilizaron los descriptores musculación, entrenamiento y obesidad. Resultados y discusión: Se obtuvieron inicialmente 596 publicaciones, siendo 11 artículos seleccionados al final para la construcción de la revisión. La mayoría de los artículos seleccionados presentaron diversos beneficios de la práctica del entrenamiento resistido en practicantes obesos, como ganancia de masa magra, pérdida de peso y mejoras en la calidad de vida. Conclusión: Ante los datos presentados es posible observar los diversos beneficios para la salud de los practicantes obesos. Estos resultados revelan la necesidad de programas de promoción y prevención de la salud que alcancen a toda la población, incluidas las personas con sobrepeso y obesidad.

Palabras clave: Entrenamiento, Obesidad, Musculación.

\section{INTRODUÇÃO}

A obesidade é considerada um problema de saúde pública, caracterizada pelo excesso de gordura no corpo, acarretando inúmeros prejuízos para saúde mundial. Além dos problemas fisiológicos, é possível associar o excesso de peso com problemas sociais, como os "corpos perfeitos" divulgados pelas mídias e diversos fatores psicológicos (MCARDLE WD, et al., 2003; FERREIRA S, 2006; WHO, 2011). A obesidade tem como origem múltiplos fatores, envolvendo aspectos ambientais e genéticos, apresentando elevada prevalência nos países em desenvolvimento (DELBIM LR e BACIUK EP, 2012).

Dados da Organização Mundial de Saúde (OMS) apontam que mais de 1,4 bilhões de adultos no mundo estão acima do peso recomendado, e mais de 500 milhões são considerados obesos (WHO, 2014). Segundo Matsudo MS, et al. (2004) a inatividade física é um dos principais fatores contribuintes pela elevada prevalência de obesidade mundial.

O exercício físico e a musculação aparecem como possibilidades para a diminuição do excesso de peso sem o consumo de medicamentos (FLECK SJ e KRAEMER WJ, 2006). Atualmente a musculação é uma das práticas esportivas que se faz mais presente no cotidiano das pessoas, devido ao crescimento das academias (BASSO CA e FERRARI HG, 2014). Uma das estratégias para o tratamento da obesidade é a realização de atividade física regular, visando o gasto calórico, e o aumento de massa magra com os treinamentos resistidos, além do controle dos níveis séricos de triglicerídeos e colesterol, sendo eles responsáveis por diversas doenças quando aumentados (WILHELMS F e NAVARRO AC, 2013).

A realização de atividade física acarreta inúmeros benefícios, como a melhora na autoestima, aumento da força e tônus muscular, associado também na diminuição da depressão (FERMINO RC, et al., 2010). A prática do treinamento resistido (exercício físico realizado com pesos adicionais, podendo ser objetos ou o próprio corpo) está associado à promoção da saúde e na prevenção de diversas condições, como diabetes, hipertensão arterial e obesidade (ARRUDA DP, et al., 2010; CALLEGARI GA, et al., 2010; ANDRADE CL e FERNANDES AR, 2011).

Embora o treinamento resistido seja eficiente nos programas de atividade física, sua prática ainda é negligenciada, havendo uma elevada recomendação por exercícios aeróbicos, inclusive para os praticantes obesos, entretanto um estudo indica que exercícios mais vigorosos tem maior eficácia para ganho de massa muscular e perda de gordura corporal (ZEMKOVÁ E, et al., 2017; KLOSTER R e LIBERALI R, 2008).

Com o crescimento da obesidade faz-se necessário a implementação de ações que incentivem a população a ter hábitos de vida mais saudáveis e possibilite o acesso a prática de atividade física para as pessoas, aumentando assim a prevenção contra doenças relacionadas ao ganho de peso corporal, auxiliando também no tratamento da obesidade (FERREIRA VA e MAGALHÃES R, 2006; ANDRELLA JL e NERY SS, 2012).

Assim, o presente estudo teve como objetivo revisar na literatura científica a respeito dos efeitos do treinamento resistido envolvendo obesos de qualquer faixa etária. 


\section{MATERIAL E MÉTODOS}

O presente estudo se caracteriza como revisão integrativa, de caráter teórico e descritivo. Foram incluídos no estudo artigos originais referentes aos efeitos do treinamento resistido em obesos, de todas as idades, publicados até dezembro de 2018. O trabalho inclui artigos quantitativos e qualitativos os quais atenderam aos seguintes critérios: (1) pesquisas com pessoas obesas, (2) musculação como exercício físico (3) pessoas de qualquer faixa etária. Foram excluídos artigos com exercícios combinados (treinamento resistido + aeróbico) ou qualquer outra atividade física que não fosse musculação, pois assim não seria possível saber os reais efeitos da musculação nos praticantes obesos. Artigos sem dados claros também foram excluídos da revisão e optou-se por não incluir teses, dissertações e monografias.

A busca foi realizada nas bases de dados eletrônicas PUBMED, PERIÓDICOS CAPES e LILACS. Os artigos que preencheram os critérios de inclusão foram avaliados independentemente do periódico. A seleção dos descritores utilizados no processo de revisão foi efetuada mediante consulta ao DeCS (Descritores em Ciências da Saúde). Para a busca dos artigos, utilizou-se uma combinação dos seguintes termos: Musculação, Treinamento e Obesidade. Todos os termos foram identificados no título e/ou resumos dos artigos. Foram utilizados recursos adicionais de acordo com a disponibilidade do banco de dados (por exemplo, Medical Subject Headings - MeSH) para amplificar a busca. As estratégias de busca estão listadas a seguir: ((Obesity [Title/Abstract]) OR adiposity [Title/Abstract])) AND (((Resistance Training [Title/Abstract]) OR Strength Training [Title/Abstract]) OR bodybuilding [Title/Abstract])). Este procedimento teve como objetivo não só filtrar os resultados, mas também cruzar os principais termos para obter o máximo de estudos possíveis. Através deste procedimento de busca, foram identificadas, inicialmente, 596 publicações (PUBMED=439, PERIÓDICOS CAPES= 149 e LILACS=9) potencialmente elegíveis para inclusão nesta revisão. No primeiro resultado dos artigos foi feita leitura e análise do título e resumos, após essa avaliação, os estudos que pareciam preencher os critérios de inclusão foram lidos na íntegra. Ao final, 11 artigos atenderam a todos os critérios de inclusão. Os principais resultados de cada artigo foram organizados em uma única tabela, com título, ano, autor, tipo de estudo e principais resultados (Tabela 1).

\section{RESULTADOS E DISCUSSÃO}

Foram encontradas inicialmente 596 publicações, na base de dados PUBMED obteve-se 439 artigos, sendo cinco artigos selecionados. Já no Periódicos CAPES, dos 149 artigos resultantes da busca, também foram selecionados cinco artigos e da base de dados LILACS dos 9 artigos resultantes da busca apenas um artigo foi selecionado. Ao final da busca 11 artigos corresponderam exatamente ao tema proposto, publicados nos seguintes periódicos: Revista Brasileira de Prescrição e Fisiologia do Exercício; Revista Brasileira de Obesidade, Nutrição e Emagrecimento; Revista Brasileira de Nutrição Esportiva; Ágora: Revista de Divulgação Científica; Pediatria Moderna; Journal Of Pakistan Medical Association; Obesity (Silver Spring); Sports; Clinical Interventions in Aging e Endotext. Os artigos foram lidos na íntegra, revisados e classificados conforme sua descrição (Tabela 1).

Dos 11 artigos incluídos nessa revisão 9 deles apresentaram o treinamento resistido como benéfico para saúde do praticante obeso e melhorias na qualidade de vida, como perda de peso (MCKEE AM e JOHN E MORLEY MB, 2018), diminuição da leptina, aumento do VO2max (Volume de Oxigênio Máximo) (YETGIN MK, et al., 2018), ganho de força muscular (SILVA AO, et al., 2018), redução de calorias (BEAVERS KM, et al., 2017), níveis de HDL (Lipoproteínas de alta Densidade) aumentados (WILHELMS F e NAVARRO AC, 2013), entre outros.

Os dados referentes aos artigos do presente estudo mostram uma possível associação do treinamento resistido com o baixo consumo de calorias nos praticantes obesos, como no estudo de McKee AM e John E Morley MB, (2018) onde os autores recomendam que os regimes de perda de peso em idosos obesos sejam associados com o treinamento resistido (musculação), corroborando com estudo de Beavers KM, (2017).

De acordo com estudo de Júnior PCFP e Ribeiro AMA, (2010), a prática do treinamento resistido ajuda a manter o metabolismo sempre ativo, contribuindo assim na prevenção de doenças associadas com a obesidade. 
Tabela 1 - Caracterização dos artigos utilizados no estudo.

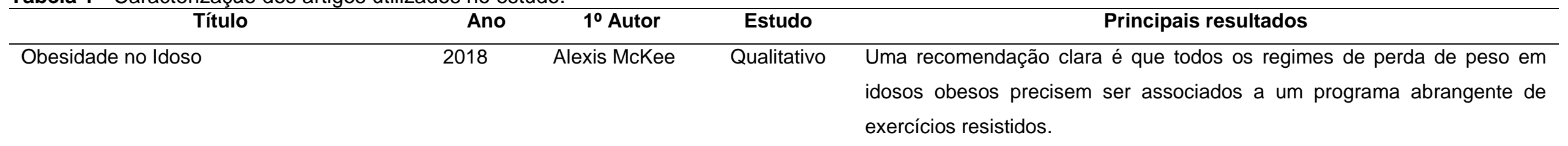

Atividade física na terapia do sobrepeso e 2018 obesidade em crianças e adolescentes. Necessidades e recomendações para programas de intervenção

Ganhos induzidos pelo treinamento de resistência na força muscular, composição corporal e capacidade funcional são atenuados em idosas com obesidade sarcopênica

Efeito do tipo de exercício durante a perda de peso intencional na composição corporal em adultos mais velhos com obesidade

Força muscular durante uma tarefa de levantamento aumenta após três meses de treinamento de resistência em indivíduos com sobrepeso e obesos

2018

2017
Meral Kucuk Descritivo, A leptina diminuiu, e o VO2max foi aumentado em ambos grupos, Yetgin quantitativo e apresentando $p$ valor significativo $<0,05$.

longitudinal

Alessandro de

Oliveira Silva

Descritivo e

longitudinal

Os resultados sugerem que as adaptações induzidas por 16 semanas de RT (treinamento resistido) são atenuadas em idosas com obesidade sarcopênica, comprometendo melhorias nos índices de adiposidade e ganhos de força muscular e capacidade funcional

Kristen M.

Beavers

Longitudinal,

controlado,

randomizado

O treinamento resistido e a redução de calorias reduziram a maior perda de peso e a alteração mais favorável na composição corporal, maximizando assim o potencial benefício funcional.

$2017 \quad$ Erika Zemková

Descritivo e

Três meses de treinamento resistido aumentaram o desempenho de longitudinal potência durante uma tarefa de levantamento modificada em indivíduos com sobrepeso e obesidade

REAS/EJCH | Vol.Sup.21 | e831 | DOI: https://doi.org/10.25248/reas.e831.2019 Página 4 de 7 


\begin{tabular}{|c|c|c|c|c|}
\hline $\begin{array}{l}\text { Consumo alimentar próximo ao treinamento } \\
\text { e avaliação antropométrica de praticantes de } \\
\text { musculação com excesso de peso em um } \\
\text { clube de Santo André-SP }\end{array}$ & 2016 & $\begin{array}{l}\text { Ruana Maia } \\
\text { Mozetic }\end{array}$ & $\begin{array}{l}\text { Descritivo e } \\
\text { transversal }\end{array}$ & $\begin{array}{l}\text { Os praticantes de musculação avaliados no presente estudo apresentaram } \\
\text { risco de doenças cardiovasculares, seja pela circunferência abdominal, pela } \\
\text { circunferência do pescoço ou pela combinação das duas. }\end{array}$ \\
\hline $\begin{array}{l}\text { Efeitos do treinamento de força sobre o } \\
\text { índice de percentual de gordura corporal em } \\
\text { adultos }\end{array}$ & 2015 & $\begin{array}{l}\text { Denis Roberto } \\
\text { Neves }\end{array}$ & $\begin{array}{l}\text { Descritivo, } \\
\text { quantitativo, } \\
\text { longitudinal }\end{array}$ & $\begin{array}{l}\text { Os resultados denotam uma melhora na qualidade de vida dos participantes } \\
\text { deste programa que contribuem firmemente para colocar a musculação ou } \\
\text { mesmo o exercício resistido como uma importante ferramenta para a perda } \\
\text { e manutenção do peso corporal. }\end{array}$ \\
\hline $\begin{array}{l}\text { Efeito de programa de treinamento resistido } \\
\text { sobre perfil lipídico e estresse oxidativo em } \\
\text { crianças pré-púberes com obesidade }\end{array}$ & 2013 & Marcelo Porto & $\begin{array}{l}\text { Descritivo e } \\
\text { longitudinal }\end{array}$ & $\begin{array}{l}\text { O programa de Treinamento Resistido (TR) foi efetivo na indução de } \\
\text { alterações benéficas nos indicadores de perfil bioquímico, incluindo lipídios } \\
\text { e glicemia e melhora da proteção do organismo contra o estresse oxidativo } \\
\text { em crianças pré-púberes com obesidade. }\end{array}$ \\
\hline $\begin{array}{l}\text { Avaliação do lipidograma e composição } \\
\text { corporal de indivíduos obesos após quatro } \\
\text { semanas de exercício de musculação } \\
\text { terapêutica }\end{array}$ & 2013 & $\begin{array}{l}\text { Francieli } \\
\text { Wilhelms }\end{array}$ & $\begin{array}{l}\text { Descritivo, } \\
\text { quantitativo, } \\
\text { longitudinal }\end{array}$ & $\begin{array}{l}\text { O treinamento mostrou tendências para melhora da composição corporal } \\
\text { (redução do percentual de gordura e aumento da massa magra), aumento } \\
\text { do colesterol total, LDL e triglicerídeos e aumento do colesterol HDL. }\end{array}$ \\
\hline $\begin{array}{l}\text { Comparar a prevalência de sobrepeso e } \\
\text { obesidade em adultos praticantes de } \\
\text { musculação }\end{array}$ & 2011 & Diane Stefan & $\begin{array}{l}\text { Descritivo e } \\
\text { transversal }\end{array}$ & $\begin{array}{l}\text { A prevalência de obesidade nos praticantes de musculação ficou abaixo do } \\
\text { esperado. As proporções de obesidade foram maiores no sexo masculino }\end{array}$ \\
\hline $\begin{array}{l}\text { Influência da musculação na prevenção da } \\
\text { obesidade }\end{array}$ & 2010 & $\begin{array}{l}\text { Paulo César } \\
\text { França Pereira } \\
\text { Júnior }\end{array}$ & Qualitativo & $\begin{array}{l}\text { Os exercícios com pesos fazem com que ocorra a diminuição do tecido } \\
\text { adiposo do indivíduo e contribui para manter o metabolismo sempre ativo, } \\
\text { mantendo o organismo em seu perfeito funcionamento, prevenindo assim } \\
\text { doenças decorrentes da obesidade }\end{array}$ \\
\hline
\end{tabular}

Fonte: Autoria dos pesquisadores, 2019.

REAS/EJCH | Vol.Sup.21 | e831 | DOI: https://doi.org/10.25248/reas.e831.2019 Página 5 de 7 
Alguns estudos ainda apresentam elevada prevalência de obesidade em praticantes de musculação, apresentando também riscos para doenças cardiovasculares (STEFAN D e LIBERALLI R, 2011; MOZETIC RM, et al., 2016). Porém, os estudos de Stefan D e Liberalli R, (2011) e Mozetic RM, et al., (2016) são estudos transversais, diferente dos estudos de Beavers KM, (2017), Wilhelms F e Navarro AC, (2013) e Neves DR, et al., (2015) que são tipos de estudos longitudinais, e acompanharam o praticante obeso por determinado tempo, sendo possível denotar melhorias na qualidade de vida dos participantes antes e depois do treinamento resistido.

Com a prática de exercícios como o treinamento resistido é possível ter alterações benéficas no perfil bioquímico (redução do percentual de gordura, diminuição da glicemia, alterações no HDL (Lipoproteínas de alta densidade) e HDL (Lipoproteínas de baixa densidade), diminuição da leptina e aumento no ganho de força), melhoras na proteção do organismo e capacidade funcional (JÚNIOR PCFP e RIBEIRO AMA, 2010; PORTO M, et al., 2013; WILHELMS F e NAVARRO AC, 2013; SILVA AO, et al., 2018; YETGIN MK, et al., 2018).

O treinamento resistido apresenta benefícios para a saúde dos praticantes de diferentes idades nos artigos selecionados, apresentando alterações benéficas no perfil bioquímico de crianças com obesidade, ganho de força e capacidade funcional em idosos com obesidade sarcopênica, benefício funcional e perda de peso em adultos mais velhos, perda e manutenção do peso corporal em adultos (PORTO M, et al., 2013; SILVA AO, et al., 2018; BEAVERS KM, et al., 2017; NEVES DR, et al., 2015). Segundo Holanda LGM, et al., (2011), a obesidade é um problema de saúde mundial, e atinge todas as faixas etárias.

Estudo de Stefan D e Liberalli R, (2011), realizado com 281 praticantes de musculação $(55,52 \%$ do sexo masculino) com média de idade de 34,64 \pm 8,01 apresentou maiores proporções de obesidade em adultos do sexo masculino, indicando índices "normais" de IMC (índice de massa corporal) nas mulheres e "sobrepeso" entre os homens. Mais de $13 \%$ dos praticantes do sexo masculino apresentavam obesidade, já o sexo feminino não chegava em $5 \%$ do total das mulheres. Essas diferenças podem estar em como eles se descocam para o trabalho, onde $12 \%$ das mulheres dizem ir trabalhar a pé, entretanto nenhum dos homens fazem esse mesmo tipo de deslocamento, sendo que quase $86 \%$ vão para o trabalho de carro ou moto. Quando questionados por quais motivos frequentavam a academia, obteve maior percentual na busca de saúde, emagrecimento e estética entre as mulheres, e os homens apresentavam como maior interesse o condicionamento físico.

Os ganhos com o treinamento resistido nos praticantes obesos aparecem na maioria dos artigos selecionados, mostrando assim a efetividade da musculação nos praticantes, oferecendo diversos benefícios para saúde e qualidade de vida. As limitações do estudo incluem uma pequena quantidade de artigos selecionados para o tema proposto, embora a maioria dos artigos presentes na literatura trabalhavam treinamento resistido combinado com os exercícios aeróbicos, sendo impossível saber de qual deles seriam os benéficos obtidos nos resultados. Os artigos selecionados também não apresentavam quantidade adequada de dias e horas para o exercício resistido em praticantes obesos, sendo necessário ressaltar a importância de cada praticante ter um treino próprio, visando seus principais interesses, desde qualidade de vida até ganho de força, e ser acompanhado por um profissional de educação física, o qual garante os cuidados fundamentais com praticantes obesos.

\section{CONCLUSÃO}

Diante dos dados apresentados é possível observar os diversos benefícios para a saúde de obesos praticantes de musculação, entre eles o ganho de massa magra, a perda de peso, a melhora no condicionamento físico, os ganhos na capacidade funcional, entre outros. Embora seja possível observar várias vantagens na prática da musculação, outras medidas podem ser elaboradas para um maior ganho na saúde desses praticantes, como associação da alimentação orientada por nutricionista com regime de perda de peso. Esses resultados revelam a necessidade de programas de promoção e prevenção da saúde que alcancem toda população, inclusive pessoas com sobrepeso e obesidade, sendo desenvolvido também programas específicos para todas as idades (adolescentes, adultos e idosos). 


\section{AGRADECIMENTOS}

Agradecemos à CAPES pela concessão de Bolsas.

\section{REFERÊNCIAS}

1. ANDRADE CL, FERNANDES AR. Treinamento com pesos e promoção da saúde em adultos: uma revisão sistemática da literatura nacional acerca dos métodos empregados em estudos científicos. Rev. Colloquium Vitae, 2011; 3(2): 5966.

2. ANDRELLA JL, NERY SS. Treinamento resistido para populações especiais: relação entre benefício e agravo patológico. Revista Funec Científica Multidisciplinar, 2012; 1(2).

3. Arruda DP, et al. Relação entre treinamento de força e redução do peso corporal. Revista Brasileira de Prescrição e Fisiologia do Exercício, 2010; 4(24).

4. BASSO CA, FERRARI HG. Percepção subjetiva de esforço como ferramenta no monitoramento da intensidade de esforço em aulas de ciclismo indoor. Revista Brasileira de Prescrição e Fisiologia do Exercício, 2014; 8(44): 149-155.

5. BEAVERS KM, et al. Effect of exercise type during intentional weight loss on body composition in older adults with obesity. Obesity, 2017; 25(11): 1823-1829.

6. CALLEGARI GA, et al. Perfil antropométrico dos indivíduos iniciantes na prática da musculação. Revista Brasileira de Prescrição e Fisiologia do Exercício, 2010; 4(24): 618-624.

7. DELBIM LR, BACIUK EP. Sobrepeso, obesidade e ônus urbano: projeções pandêmicas ou sensacionalismo? Intellectus. Rev Acadêmica Digital das Faculdades Unopec, 2012; 8(2): 28-43.

8. FERMINO RC, et al. Motivos para prática de atividade física e imagem corporal em freqüentadores de academia. Rev. Bras. Med. Esporte, 2010; 16(1).

9. FERREIRA S, et al. Aspectos etiológicos e o papel do exercício físico na prevenção e controle da obesidade. Revista de Educação Física, 2006; 133: 15-24.

10. FERREIRA VA, MAGALHÃES R. Obesidade no Brasil: tendências atuais. Revista Portuguesa de Saúde Pública, 2006; 24(2).

11. FLECK SJ, KRAEMER WJ. Fundamentos do treinamento de força muscular. Porto Alegre: Artmed, $2006 ; 19$.

12. HOLANDA LGM, et al. Excesso de peso e adiposidade central em adultos de Teresina-PI. Revista da Associação Médica Brasileira, 2011; 57(1).

13. JÚNIOR PCFP, RIBEIRO AMA. Influência da musculação na prevenção da obesidade. Ágora: Revista Divulgada Cientificamente, 2010; 17(2).

14. KLOSTER R, LIBERALI R. Emagrecimento: composição da dieta e exercício físico. Revista Brasileira de Nutrição Esportiva, 2008; 2(11): 288-306.

15. MATSUDO SM, et al. Atividade física no tratamento da obesidade. Einsten, $2004 ; 4(1): 29-43$.

16. MCARDLE WD, at al. Fisiologia do Exercício: Energia, Nutrição e Desempenho Humano. 5. ed. Rio de Janeiro: Guanabara Koogan. 2003.

17. MCKEE AMD, JOHN E MORLEY MB. Obesity in the Elderly. Context, 2018.

18. MOZETIC RM. et al. Consumo alimentar próximo ao treinamento e avaliação antropométrica de praticantes de musculação com excesso de peso em clube de Santo André-SP. RBNE-Revista Brasileira de Nutrição Esportiva, 2016; 10(55): 31-42.

19. NEVES DR, et al. Efeitos do treinamento de força sobre o índice do percentual de gordura corporal em adultos. RBONERevista Brasileira de Obesidade, Nutrição e Emagrecimento, 2015; 9(52): 135-141.

20. PORTO M, et al. Efeito de programa de treinamento resistido sobre perfil lipídico e estresse oxidativo em crianças prépúberes com obesidade. Pediatr, 2013; 49(10).

21. SILVA AO, et al. Resistance training-induced gains in muscle strength, body composition, and functional capacity are attenuated in elderly women with sarcopenic obesity. Clin Interv Aging, 2018; 13: 411- 417. Doi: 10.2147/CIA.S156174

22. STEFAN D, LIBERALI R. Comparar a prevalência de sobrepeso e obesidade em adultos praticantes de musculação. RBONE-Revista Brasileira de Obesidade, Nutrição e Emagrecimento, 2012; 5(29).

23. WHO. Obesity: preventing and managing of WHO Consulation on Obesity. Geneva: Wolrd Healt Organization. 2011.

24. WHO. World Health Organization. Obesity and overweight. 2014. Disponível em: http://www.who.int/mediacentre/ factsheets/fs311/en/

25. WILHELMS F, NAVARRO AC. Avaliação do lipidograma e composição corporal de indivíduos obesos após quatro semanas de exercício de musculação terapêutica. Revista brasileira de prescrição e fisiologia do exercício, 2013; 7(39): 260-267.

26. YETGIN MK, et al. The influence of physical training modalities on basal metabolic rate and leptin on obese adolescent boys. Journal of the pakistan medical association, 2018; 68(6): 929-931.

27. ZEMKOVÁ E, et al. Muscular power during a lifting task increases after three months of resistance training in overweight and obese individuals. Sports, 2017; 5(2): 35. 